\title{
Vergleichende Betrachtungen zur Bestimmung des asymptotischen Verhaltens mehrdimensionaler Laplace-Gauß-Integrale
}

\author{
H. Birndt und'W.-D. Richter
}

Für gewisse in der Theorie der Wahrscheinlichkeiten großer Abweichungen interessierende Borelmengen mit gegen Unendlich strebendem Abstand vom Koordinatenursprung wird das asymptotiśche Verhalten ihres Gaußmaßes untersucht. Dazu werden verschiedene Methoden des Studiums von Laplace-Gauß.Integralen miteinander verglichen. Einige neue Aussagen werden durch zahlreiche Beispiele illustriert.

Изучается асимптотическое поведение гауссовской меры для некоторых боре́левских множеств, интересующих в теории вероятностей больших уклоневия и расстояние которых от начала координат стремится к бесконечности. Для әтого сравниваются различные методы изучения интегралов Лапласа-Гаусса. Некоторые новые результаты иллюстрируются разными примерами.

The asymptotic behaviour of the Gaussian measure is investigated for some Borel sets in which one is interested in the theory of probabilities of large deviations and the distance of which from the origin tends to infinity. To that end, various methods for studying LaplaceGaussintegrals are compared. Some new assertions are illustrated by many examples.

\section{Einleitung}

In der Summationstheorie zufälliger Vektoren und insbesondere beim Studium sogenannter großer Abweichungen entsteht die Frage nach dem asymptotischen $(x \rightarrow \infty \infty)$. Verhalten der Wahrscheinlichkeit $P\left(Z_{B} \in x A\right)$ dafür, daß ein Gauß'scher Zufallsvektor $Z_{B}$ mit Mittelwert $\mathfrak{v}=(0, \ldots, 0) \in \mathbf{R}^{k}(k \geqq 2)$ und regulärer Kovarianzmatrix $B$ in die Menge $x A=\left\{\left(x z_{1}, \ldots, x z_{k}\right): \ddot{z}=\left(z_{1}, \ldots, z_{k}\right) \in A\right\}$ fällt, wobei $A$ einer gewissen Klasse. $\mathfrak{S}$ von Borelmengen angehört. Bezeichnet $\Phi(\cdot)$ die Verteilung von $Z_{B}$ und $B^{-1}$ die Inverse von $B$, so gilt

$$
\begin{aligned}
P\left(Z_{B} \in x A\right) & =\Phi(x A) \\
& =x^{k} \int_{A}\left((2 \pi)^{k} \operatorname{det} B\right)^{-1 / 2} \exp \left\{-\frac{x^{2}}{2} z B^{-1} z^{T}\right\} d z .
\end{aligned}
$$

Dieses Integral wollen wir als Laplace-Gauß-Integral bezeichnen. Dem .Studium solcher und wesentlich allgemeinerer Laplace-Integrale haben sich zahlreiche Autoren (u. a. $[1-7,9]$ ) gewidmet. In der. vorliegenden Arbeit sollen verschiedene sowohl bekannte als auch neue Methoden des Studiums von Laplace-Gauß-Integralen miteinander verglichen werden. Im Zusammenhang mit der Arbeit [7] ${ }^{1}$ ) wird hierbei ein Úberblick über derzeit praktizierte Vorgehensweisen angestrebt.

1) Das ist der vorangegangene Beitrag dieses Heftes. 
In'Abschnitt 2 wollen wir zunächst einige grundlegende Aussagen über das grobe Verhalten von $\Phi(x A)$, d. h. über das Verhalten von $\ln \Phi(x A)$ für $x \rightarrow \infty$ darstellen, welche aus den Arbeiten von V. P. Maslov und.M. V. FedorJuk [5: Satz 1] und W.-D. Richter [6: Theorem 1] folgen. Daran schließen sich dann genauere Betrachtungen über $\Phi(x A)$ an: Im Abschnitt 3 werden die Untersuchungen aus [5: Satz 2] fortgefüht, und insbesondere wird eine $\operatorname{der}$ in [5] an den Rand $\partial A \operatorname{der}$ Menge $A$ gestellten Bedingungen (siehe (6) unten) diskutiert. Die Ordnung, mit der $\Phi(x A)$ für $\stackrel{\prime}{x} \rightarrow \infty$ gegen Null strebt, kann aus den Resultaten dieses Abschnittes auch noch nicht voliständig bestimmt werden, jedoch schon wesentlich besser als im Abschnitt 2. - Im Abschnitt 4 schließlich wird eine Anwendung der Resultate der klassischen' Laplace-Methode (siehe z. B. die Monographie [4] von M. V. FedorJuk) demonstriert. Unter Ausnutzung in allgemeinen schärferer Voraussetzungen als in 'den vorangegangenen Abschnitten können sowohl die Ordnung von $\Phi(x A)$ für $x \rightarrow \infty$ als auch die zugehörige asymptotisch echte Konstante exakt bestimmt werden. Im Hinblick auf Anwendungen in der Wahrscheinlichkeitstheorie werden diese Betrachtungen sofort für den Fall durchgeführt, daß die Kovarianzmatrix $B$ von $x$ abhängen kann, jedoch für $x \rightarrow \infty$ gegen eine reguläre Matrix strebt.

Es sei darauf hingewiesen, da $B$ die in der Arbeit.[7] dargelegte Methode es prinzipiell gestattet, unter den vergleichsweise schwächsten Voraussetzungen an $\partial A$ die Genauigkeit der mit der Laplace-Methode erzielten Aussagen zu erreichen. Das gelingt, indem das Ausgangsproblem auf geeignete elementar-geometrische Betrachtungen zurückgeführt wird (siehe auch [9]).

Zum Schluß dèr Einleitung führen wir noch einige Bezeichnungen ein. Es seien $S=\left\{z \in \mathbf{R}^{k}:\|z\|=1\right\}$ die Oberfläche der Einheitskugel bezüglich der Euklidischén Norm $\|\cdot\| ; \dot{A}$ die Abschließung, $\not$ das Innere und $A^{c}$ das Komplement einer Menge $A \subset \mathbf{R}^{k}$. Zu jedem. Punkt $z \in \mathbf{R}^{k}$ mit $\|z\|=r>0$ existiert' bekanntlich ein eíndeutig. -bestimmtes $f=\left(f_{1}, \ldots, f_{k-1}^{\prime}\right)$ aus $\mathfrak{B}:=\left(\stackrel{k-2}{X}[0, \pi)_{1}(0,2 \pi)\right.$ derart, daß gilt $\dot{z} / r=\left(\cos f_{1}, \sin f_{1} \cos f_{2}, \ldots, \sin f_{1} \ldots \sin f_{k-1}\right)$. Die Schreibweise $z=z[f, r] \stackrel{c}{=} r \cdot z[f, 1]$ symbolisiert also die Darstellung eines Punktes durch verallgemeinerte Kugelkoordinaten. Im weiteren wollen wir sagen, daß eine Menge $A$ zu einer Teilklasse $\subseteq \mathfrak{B}$ von 、 Borelmengen mit "glattem Rand" gehört, wenn sie die Eigenschaften ( $\subseteq \mathfrak{B} 1)$ bis ( $\subseteq \mathfrak{B} 3$ ) besitzt:

$$
\mathfrak{o} \in A^{c} \text {. }
$$

Auf einer (eindeutig bestimmten) endlichen Vereinnigung $\mathfrak{B}(A)$

I offener Teilintervalle von $\mathfrak{B}$ ist eine Funktion $R_{A}$ so definiert, daß gilt

$$
\stackrel{A}{=}=\left\{z[f, r]: r>R_{A}(f), f \in \mathfrak{B}(A)\right\} \text {. }
$$

$R_{A}{ }^{\prime}$ ist positiv und-stetig auf $\overline{B(A)}$ und besitzt auf $\mathfrak{B}(A)$ stetige partielle Ableitungen erster Ordnung bezüglich aller Argunente.

Diese Klasse wurde unter anderem in [6] und [8] betrachtet. Im Falle $\mathfrak{B}(A)=\mathscr{B}$ beinhaltet $(\subseteq \mathfrak{B} 3$ ) die Bedingung $(B 1)$ aus [6]. Wir wollen auch die dort eingeführte Bezeichnungsweise

$$
z(f, r)=z\left[f, R_{A}^{\prime}(f) r\right]
$$

verwenden. 


\section{Grobe Aussagen}

Es seien

$$
\dot{H}(f)= \begin{cases}\left(\sin f_{1}\right)^{k-2} \ldots \sin f_{k-2} & \text { für } k \geqq 3 \\ 1 & \text { für }, k=2\end{cases}
$$

die Funktionaldeterminante der den Utbergang von verallgemeinérten Kugelkoòrdinaten zu kartesischen'Koordinaten vermittelnden Abbildung,

$$
\begin{aligned}
& g(f)=z(f, 1) B^{-1} z^{T}(f, 1), \quad f \in \mathfrak{B}(A), \\
& h(f)=\left(R_{A}(f)\right)^{k}|H(f)| / g(f), \quad f \in \mathfrak{B}(A), \\
& \theta_{0}=\int_{\mathscr{B}(A)} h(f) d f, \\
& F(x)=\int_{\mathfrak{B}(A)} h(f) \exp \left\{-\frac{x^{2}}{2} g(f)\right\} d f
\end{aligned}
$$

und

$$
a=\inf \left\{z B^{-1} z^{T}: z \in A\right\} .
$$

Aus den Resultaten in [6] folgt für $A \in \subseteq \mathfrak{B}$

$$
\Phi(x A)=\left((2 \pi)^{k} \operatorname{det} B\right)^{-1 / 2} x^{k-2} F(x)\left(1+O\left(1 / x^{2}\right)\right), \quad x \rightarrow \infty .
$$

Im Falle der zusätzlichen Erfüllung der (zur Bedingung $(B$ 2) aus [6] äquivalenten) , Bedingung

$$
\mathfrak{B}_{0}^{\prime}=\{f \in \mathfrak{B}(A): g(f)=a\} \text { hat das Lebesguema } ß \text { Null in } \mathfrak{B}(A)
$$

gilt überdies $F(x)=o(1) \exp \left\{-x^{2} a / 2\right\}, x \rightarrow \infty$. Das legt es nahe, (1) in der Form

$$
\Phi(x A)=\left((2 \pi)^{k} \operatorname{det} B\right)^{-1 / 2} 0_{0} x^{k-2} \exp \left\{-x^{2} d(x) / 2\right\}\left(1+O\left(1 / x^{2}\right)\right)
$$

zu schreiben, wobei die Funktion $d$ als Lösung der Gleichung

$$
\theta_{0} \exp \left\{-x^{2} d(x) / 2\right\}=F(x)
$$

definiert, ist. Sie genügt den Ungleichungen.

$$
a \leqq d(x) \leqq a+2\left(\gamma+\ln \theta_{0}-\ln \theta_{1}(\gamma)\right) / x^{2}
$$

für beliebige $x>0, \gamma>0$, wobei $\theta_{1}(\gamma)=\int h(f) d f$ und das Integrationsgebiet des letzten Integrales $\mathfrak{B}_{2 \gamma / x^{*}}$ ist, mit

$$
\mathfrak{B}_{c}=\{f \in \mathfrak{B}(A): a \leqq g(f) \leqq a+c\}, \quad c \geqq 0 .
$$

Außßerdem gilt unter den Voraussetzungen $A \in \subseteq \mathfrak{E}$ und (B 2) für $x \rightarrow \infty$ die Beziehung $x^{2}(d(x)-a) \rightarrow \infty$. Aus (1), (3) und (4) läßt sich unschwer ableiten, daß für $A \in \subseteq \mathfrak{B}$ gilt [5]

$$
\lim _{x \rightarrow \infty} 2 \ln F(x) / x^{2}=-a
$$

\section{Präzisierungen}

Die im folgenden aus technischen Grüden verwendete Voraussetzung

$$
H\left(f_{0}\right) \neq 0, \quad f_{0} \in B_{0}
$$

läßt sich gegebenenfalls durch eine geeignete Umorientierung des zugrunde gelegten . Koordinatensystems erfüllen, insbesondere im Falle der Erfüllung der weiter unten 
getroffenen Voraussetzung

$$
\text { card } \mathfrak{B}_{0}=N<\infty \text {. }
$$

Mit $\mu^{(k)}$ bezeichnen wir das LebesguemaB im $\mathbf{R}^{k}$ und setzen $V(c)=\mu^{(k-1)}\left(\mathfrak{B}_{c}\right)$. Aus [5] folgt, daß für $A \in \mathfrak{S} \mathfrak{B}$, unter den Voraussetzungen ( $\mathfrak{B} 3$ ) und

$$
\text { es existiert } \lim _{c \rightarrow 0} \ln V(c) / \ln c=: \alpha>0
$$

für $x \rightarrow \infty$ gilt:

$$
\ln F(x)=-a x^{2} / 2-\alpha \ln \left(x^{2} / 2\right)+o(1) \ln \left(x^{2} / 2\right) .
$$

Hieraus und aus (1) folgt somit, daß unter den Voraussetzungen $A \cdot \in \mathfrak{S} \mathfrak{B}$, ( $\mathfrak{B} 3$ ) und (6) für $x \rightarrow \infty$ gilt

$$
\Phi(x A)=x^{k-2(1+a)+o(1)} \exp \left\{-a x^{2} / 2\right\} \text {. }
$$

Sieht man sich den Beweis der Aussage (7) in [5] an, so entsteht der Eindruck, da B auf der Grundlage der dort verwendeten Methode eine weitere Präzisierung der Konvergenzordnung in.(8) nur schwer zu erreichen sein wird. Zur anschaulichen Interpretation der Bedingung (6) verweisen wir auf eine korrespondierende Bedingung in [8: Theorem 1]. Wir bemerken, daß (6) die Erfüllung der Bedingung ( $(92)$ nach sich zieht, weil im Falle der Verletzung von $(\mathfrak{B} 2)^{\prime}$ nämlich $\alpha=0$ gilt. Da aus [5] keine hinreichend allgemeinen Bedingungen an $\partial A$ ableitbar. sind, inter denen die Voraussetzung (6) erfüllt ist, wollen wir uns im folgenden dieser Frage widmen. Dazu fïhren wir zunächst die folgende Verschärfung der Voraussetzung (SB 3) ein:

In $\mathfrak{B}(A)$ existiert die Matrix $g^{\prime \prime}(f)$ stetiger gemischter partieller Ableitungen zweiter Ordnung der Funktion $g(f)$, und es ist $g^{\prime \prime}\left(f_{0}\right)$ positiv definit für alle $f_{0} \in \mathfrak{B}_{0}$.

(Im Falle $k=2$ handelt es sich bei $g^{\prime \prime}(f)$ natürlich einfach um die zweite Ableitung der Funktion $g(f), f \in(0 ; 2 \pi)$.),

Satz 1: Für jede Menge $A \in \subseteq \mathfrak{G}$, welche den Voraussetzungen ( $\mathfrak{B}$ 4) und.(B-5) genügt, existiert der Grenzwert (6), und es gilt $\alpha=(k-1) / 2$. Es ist also für $A \in \mathfrak{S} \mathfrak{B}$. mit $(\mathfrak{B} 3)-(\mathfrak{B} 5)$

$$
\Phi(x A)=x^{-1+o(1)} \exp \left\{-a x^{2} / 2\right\}, \quad, x \rightarrow-\infty .
$$

Beweis: Es sei zunächst $N=1$. Das einzigè Element von $\dot{\mathfrak{B}}_{0}$ bezeichnen wir mit $f_{0}$. Wegen grad $g\left(f_{0}\right)=\mathrm{o}$ gilt

$$
g(f)=g\left(f_{0}\right)+\left(f-f_{0}\right) g^{\prime \prime}\left(f_{0}\right)\left(f-f_{0}\right)^{T} / 2+\delta(1)\left\|f-f_{0}\right\|^{2}
$$

für $f \rightarrow f_{0}$. Im Falle $c \rightarrow 0$ streben wegen der Stetigkeit der Funktion $g$ alle $f \in \mathfrak{B}_{c}$ zu $f_{0}$. Es gilt dann also

$$
\mathfrak{B}_{c}=\left\{f \in \mathfrak{B}(A): 0 \leqq\left(f-f_{0}\right)\left[g^{\prime \prime}\left(f_{0}\right)+o(1) I\right]\left(f-f_{0}\right)^{T} / 2 \leqq c\right\}
$$

mit $I=\left(\begin{array}{ccc}1 & \ldots & 1 \\ \vdots & & \vdots \\ 1 & \ldots & 1\end{array}\right)$. Das hei $\beta \mathrm{t}, V(c) \asymp c^{\alpha}$.

Im Falle $N>1$ folgt die Behauptung des Satzes unmittelbar aus der Beziehung $\ln (N V(c)) / \ln c \sim \ln V(c) / \ln c \rightarrow \alpha, c \rightarrow 0$ 
Aus àllem Gesagten wird ersichtlich, daß für das Vorliegen der Beziehung (6) (und damit (7)) die Eigenschaften der Menge $\mathfrak{B}_{0}$ eine wichtige Rolle spielen. Der folgende Satz widmet sich einer weiteren diesbezüglichen Charakterisierung.

Satz 2: Es sei $k \geqq 3$, und die Menge $A \in \mathfrak{S B}$ genüge der Voraussetzung (B 5). Für ein $r \in\{1, \ldots, k-2\}$ und alle $c \geqq 0$ gelte $\mathfrak{B}_{c}=\mathfrak{B}_{c}(r) \times \mathfrak{B}_{c}(k-1-r)$, wobei $\mu^{(k-1-r)}\left(\mathfrak{B}_{0}(k-1-r)\right)>0$. und card $\mathfrak{B}_{0}(r)<\infty$ ist. Außerdem seien die kleinsten Eigenwerte der Matrizen $\left(\frac{\partial^{2}}{\partial f_{i} \partial f_{j}} g(f)\right)_{1 \leqq i, j \leqq r}$ für alle $\left(f_{r+1}, \ldots, f_{k-1}\right) \in \mathfrak{B}_{0}(k-1-r)$ gleichmäßig nach unten durch eine positive Konstante beschränkt. Dann existiert $\alpha=\lim _{c \rightarrow 0} \ln V(c) / \ln c$, und es gilt $\alpha=r / 2$. Es ist also für $A \in \subseteq \mathfrak{B}$ mit $(\mathfrak{B} 3)-($ B 5)

$$
\Phi(x A)=x^{k-2-\tau+o(1)} \exp \left\{-a x^{2} / 2\right\}, \quad x \rightarrow \infty .
$$

Beweis: Wegen $\ln \mu^{(k-1-r)}\left(\mathfrak{B}_{c}(k-1-r)\right) / \ln c \rightarrow 0$ für $c \rightarrow 0$ und $\ln V(c)$ $=\ln \mu^{(k-1-r)}\left(\mathfrak{B}_{c}(k-1 \beth r)\right)+\ln \mu^{(r)}\left(\mathfrak{B}_{c}(r)\right)$ ist das Problem auf die Aussage von Satz 1 zurückgeführt

Für die Betrachtung der folgenden Beispiele setzen wir der Einfachheit halber voraus, da $B$ mit der jeweiligen Einheitsmatrix übereinstimmt und $A \in \subseteq \mathfrak{B}$ gilt.

Beispiel' 1: Im Falle $\partial A=\left\{z \in \mathbf{R}^{2}: \max \left(\left|z_{1}\right|,\left|z_{2}\right|\right) \doteq 1\right\}$ ergibt sich $g(f)=R^{2}(f)$, $\mathfrak{B}_{0}=\{0, \pi / 2, \pi, 3 \pi / 2\}, a=1$. Wir betrachten ohne Einschränkung der Allgemeinheit im weiteren nur noch eine Umgebung von $f_{0}=\pi / 2: R(f)=1 / \sin f$ für $\pi / 4 \leqq f$ $\leqq 3 \pi / 4, g^{\prime \prime}\left(f_{0}\right)=2$. Aus Satz 1 folgt $\alpha=1 / 2$ und (9).

Beispiel 2: $\operatorname{Im}$ Falle $\partial A=\left\{z \in \mathbf{R}^{3}: \max \left(\left|z_{1}\right|,\left|z_{2}\right|,\left|z_{3}\right|\right)=1\right\}$ gilt wieder $g(f)=R^{2}(f)$, $a=1$. Wir betrachten $f_{0}=(\pi / 2, \pi / 2) \in \mathfrak{B}_{0}^{-}: R(f)=1 / \sin f_{1} \sin f_{2}$ für $\pi / 4 \leqq f_{i}$ $\leqq 3 \pi / 4, i=1,2, g^{\prime \prime}\left(f_{0}\right)=\left(\begin{array}{ll}2 & 0 \\ 0 & 2\end{array}\right)$. Aus Satz 1 folgt wiederum (9).

Beispiel 3: Es seị $\partial A \doteq\left\{z \in \cdot \mathbf{R}^{3}:-2 \leqq z_{1} \leqq 2, z_{2}{ }^{2}+z_{3}{ }^{2}=1\right\} \cup\left\{z \in \mathbf{R}^{3}:\left|z_{1}\right|=2\right.$, $\left.z_{2}{ }^{2}+z_{3}{ }^{2} \leqq 1\right\}$. Dann ist $\mathfrak{B}_{0}=\{\pi / 2\} \times[0,2 \pi), a=1$ und $g(f)=1$ für $f \in \mathfrak{B}_{0}$. Ist $f_{1} \neq \pi / 2$ und $\left|f_{1}-\pi / 2\right|<\varepsilon$ für ein $\varepsilon>0$, so wird $g(f)^{\prime}=R^{2}(f)=\left(\sin f_{1}\right)^{-2}, 0 \leqq \mathrm{f}_{2}$ $<2 \pi$. Weiter gilt $g^{\prime \prime}\left(\pi / 2, f_{2}\right) \doteq 2$ für alle $f_{2} \in[0,2 \pi)$. Nach Satz $2 \backslash$ folgt mit $r=1$, $\operatorname{da} \beta \Phi(x A)=x^{o(1)} \exp \left\{-x^{2} / 2\right\}, x \rightarrow \infty$ ist.

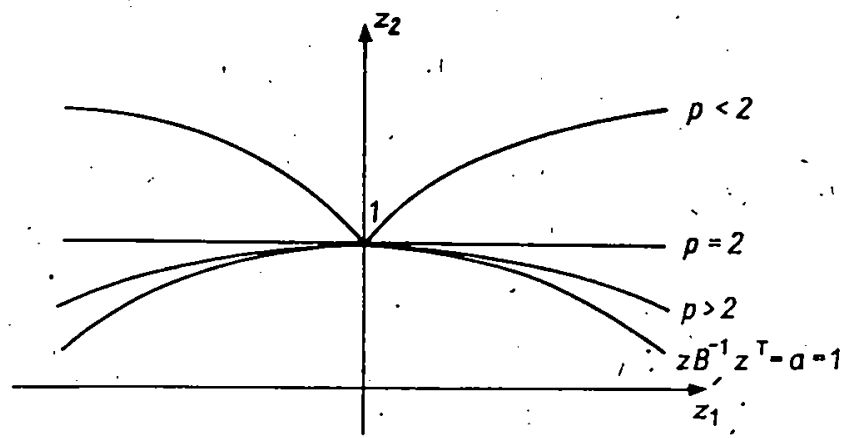

Abb.: Verlauf von. $\partial A=\left\{\left(z_{1}, z_{2}\right) \in \mathbf{R}^{2}: z_{1}{ }^{2}+z_{2}{ }^{2}=1+\left|z_{1}\right|^{p}\right\}$ in $U=U((0 ; 1))$ für verschiedeno $p . \dot{>} 0$.

Beispiel 4 (s. Abb.): Sei $N=1$, und für eine Umgebung $U=U((0,1))$ des Punktes $(0,1)$ gelte $U\left\ulcorner\partial A \subseteq \mathfrak{M}_{p}=\left\{\left(z_{1}, z_{2}\right) \in \mathbf{R}^{2}: z_{1}{ }^{2}+z_{2}{ }^{2}=1+\cdot\left|z_{1}\right|^{p}\right\}\right.$ für ein $p>0$. Bezeichnet $\left(z_{1}^{c}, z_{2}^{c}\right)$ den Schnittpunkt der Kurven $\mathfrak{M}_{p}$ und $\left\{\left(z_{1}, z_{2}\right) \in \mathbf{R}^{2}\right.$ : 
$\left.\dot{z} B^{-1} z^{T}=1+c\right\}$ fïr $z_{1}^{c}>0, z_{2}^{c}>0$, so ergibt sich $\dot{V(c)}=2 \arctan \left(z_{1}^{c} / z_{2}{ }^{c}\right)$ und $\alpha=\lim _{c \rightarrow 0} \ln V(c) / \ln c=\lim _{c \rightarrow 0} \ln \left(2 \arctan \left\{c^{1 / p} /\left(1-c^{2 / p}+c\right)^{1 / 2}\right\}\right) / \ln c=1 / p$. Das hei $\beta \mathrm{t}$, es gilt

$$
\Phi(x A)=x^{-2 / p+o(1)} \exp \left\{-x^{2} / 2\right\}, \quad x \rightarrow \infty .
$$

\section{Weitere Präzisierungen mittels der Laplace-Methode}

Eigentlich stellt ( $(B)$ ) aus Abschnitt 3 bereits eine für die Laplace-Methode typische Bedingung.dar. In den Sätzen 1 und 2 ist die in ( $(B 5)$ enthaltene Information jedoch noch nicht hinreichend ausgewertet. Dies soll in Satz 3 geschehen.

Wir betrachten eine Familie $\{A(x)\}_{x>0}$ von Mengen $A(x)$ aus $\bigodot \mathfrak{B}$ und eine Familie $\{B(x)\}_{x>0}$ von symmetrischen und regulären Matrizen, die im Hinblick auf wahrscheinlichkeitstheoretische Anwendungen die Eigenschaft

$$
B(x)^{-1}=(1+o(1)) B^{-1}, \quad x \rightarrow \infty
$$

besitzen sollen, wobei $B$ eine symmetrische, positiv definite Matrix ist. Die in den Abschnitten 1 und 2 eingeführten Bezeichnungen $R_{A}(f), z(f, r), g(f), h(f), a, \mathfrak{B}_{0}$ und $\mathfrak{B}(A)$ verwenden wir im folgenden völlig sinngemä $\beta$ für jede Menge $A(x) \in \cong \mathfrak{V}$ und versehen sie zur Únterscheidbarkeit nit dem Index $x: h_{x}(f), z_{x}(f, r), g_{x}(f), h_{x}(f)$, $a_{x}, \mathfrak{B}_{0 x}$ und $\mathfrak{B}_{x}(A(x))$. Mit Hilfe dieser (xrößen formulieren wir nun weitere Voraussetzungen.

$$
\begin{aligned}
& H\left(f_{0 x}\right) \neq 0 \text { für alle } f_{0 x}^{\prime} \in \mathfrak{B}_{0 x} \text { und alle } x>0 . \\
& \text { card } \mathfrak{B}_{0 x}=N<\infty \text { für alle } x>0 .
\end{aligned}
$$

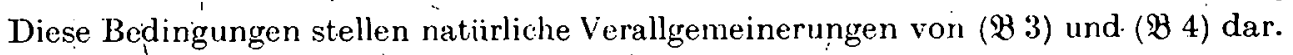

Es gibt ein $\varepsilon>0$ so, dabin $U\left(\mathfrak{V}_{0 x}\right)=\left\{f \in \mathfrak{B}_{x}(A(x)):\left\|f-f_{0 x}\right\|<\varepsilon\right.$ fiir ein $\left.f_{0 x} \in \mathscr{B}_{0 x}\right\} \subseteq \mathfrak{B}_{x}(A(x))$ die Matrix $g_{x}{ }^{\prime \prime}(f)$ der partiellen $\mathrm{Ab}$ leitungen zweiter Ordnung der Funktion $g_{x}(f)$ existiert für alle $x>0$.

Es seien $\delta$ und $\varrho$ reelle Funktionen auf $[0, \infty)$ mit $\delta(x) \rightarrow 0, \varrho(x) \rightarrow \infty$ und $\delta^{2}(x) \varrho(x)$ $\stackrel{\rightarrow}{\rightarrow}$ für $x \rightarrow \infty$. Für jedes $x>0$ bezeichne $\lambda_{x}(f)$ den kleinsten Eigenwert der Matrix $g_{x}{ }^{\prime \prime}(f), f \in U\left(\mathcal{B}_{0 x}\right)$. (Im Falle $k=2$ gilt $\lambda_{x}(f)=g_{x}{ }^{\prime \prime}(f)$.)

$$
\lambda_{x}(f) \geqq \varrho(x) \ln x / x^{2}, / \text { für alle } f \in \mathfrak{B}_{0 x}, \quad x \geqq x_{0} .
$$

Es seien $\tilde{g}_{x}(f)$ die symmetrische, positiv definite Matrix mit

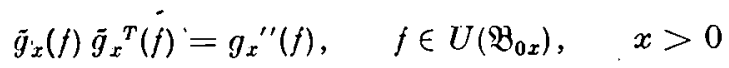

und

$$
Q\left(f_{0 x}\right)=\left\{f \in \mathfrak{B}_{x}(A(x)):\left\|\left(f-f_{0 x}\right) g_{x}(f)\right\| \leqq \lambda_{x}(f)^{1 / 2} \delta(x)\right\}
$$

für $f_{0 x} \in \mathfrak{B}_{0 x}, x>0$.

Für alle $f_{0 x} \in \mathfrak{B}_{0 x}$ gilt gleichmäßig bezüglich $f \in \Omega\left(f_{0 x}^{-}\right)$

$$
g_{x}^{\prime \prime}(f)=(1+o(1)) g_{x}{ }^{\prime \prime}\left(f_{0 x}\right), \quad x \rightarrow \infty .
$$

Die Bedingungen $(\mathfrak{L})-(\mathfrak{Q} 6)$ gemeinsam verallgemeinern $(\mathfrak{B} 5)$.

$$
\dot{g}_{x}(f) \geqq a_{x}+2(k+2) \ln x / x^{2} \quad \text { für } \quad f \in \mathfrak{B}_{x}(A(x)) \backslash U\left(\mathfrak{B}_{0 x}\right), \quad x>0 .
$$


Zur Veranschaulichụg dieser Bedingung sei an das oben angeführte Beispiel 4 erinnért. Der dort auftretende Parameter $p>0$ kann zwar beliebig groß sein; darf aber nicht (ohne weitere Betrachtungen anzustellen) gemeinsam mit $x$ gegen Unendlich streben. Bedingung ( $\Omega 7$ ) schließt dies jedoch in gewissen Grenzen nicht aus.

Satz 3: Genügen eine Familie $\{A(x)\}_{x>0}$ von Mengen aus $\subseteq \mathfrak{B}$ und eine Familie symmetrischer, positiv definiter Matrizen $\{B(x)\}_{x>0}$ den Bedingungen $(\Omega 1)-(\Omega(7)$, so gilt

$$
\Phi(x A(x))=\frac{1+o(1)}{\sqrt{\pi \operatorname{det} B}} 2^{k / 2-1} \sum_{2 \mathfrak{Y}_{0 x}} \frac{h_{x}\left(f_{0 x}\right)}{\sqrt{\operatorname{det} g_{x}{ }^{\prime \prime}\left(f_{0 x}\right)}} x^{-1} \exp \left\{-x^{2} a_{x} / 2\right\}, \quad x \rightarrow \infty .
$$

Aus diesem Satz folgt, daß unter sehr allgemeinen Bedingungen an $\partial A(x)$ für $x \rightarrow \infty$ die Größe $\Phi(x A(x))$ mit der gleichen Ordnung $x^{-1} \exp \left\{-x^{2} a_{x} / 2\right\}$ gegen Null strebt, wie das entsprechende Gaußmaß eines Halbraumes mit Abstand $x a_{x}^{1 / 2}$ vom Koordinatenursprung. Der Einfluß der Dimension $k$ auf die entsprechende asymptotisch echte Konstante ist in (12) explizit angegeben.

Beweis von Satz 3: Der Einfachheit halber sei $N=1, \mathfrak{B}_{0 x}=\left\{f_{0 x}\right\}$. Analog zu (1) erhält man für $x \rightarrow \infty$

$$
\Phi(x A(x))=\left((2 x)^{k} \operatorname{det} B(x)\right)^{-1 / 2} x^{k-2} F(x)\left(1+O \dot{O\left(x^{-2}\right)}\right)
$$

wobei.

$$
F(x)=\int_{\mathscr{Z}_{x}(A(x))} h_{x}(f) \exp \left\{-x^{2} g_{x}(f) / 2\right\} d f
$$

ist. Mit $I_{1 x}, I_{2 x}$ bzw. $I_{3 x}$ bezeichnen.wir Integrale mit dem gleichen Integranden und den' Integrationsgebieten $\Omega\left(f_{0 x}\right), \quad\left(\mathfrak{B}_{x}(A(x)) \backslash \Omega\left(f_{0 x}\right)\right) \cap U\left(\mathscr{B}_{0 x}\right)$ bzw.' $\left(B_{x}(A(x))\right.$ $\left.\backslash \Omega\left(f_{0 x}\right)\right) \backslash U\left(\mathfrak{B}_{0 x}\right)$. Dann gilt $F(x)=I_{1 x}+I_{2 x}+I_{3 x}$.

Aus ( $(\mathbf{Q} 7)$ folgt

und damit

$$
I_{3 x} \leqq \exp \left\{-x^{2} a_{x} / 2-(k+1) \ln x\right\} \int_{\mathbb{N}_{x}(A(x))} h_{x}(f) d f
$$

$$
I_{3 x}=O(1) x^{-1-k} \exp \left\{-x^{2} a_{x} / 2\right\}, \quad x \rightarrow \infty .
$$

- Wegen $\operatorname{grad} g_{x}(f)=\mathfrak{o}$ für ein $f \in \mathscr{B}_{0 x}$ erhält man nach Taylorentwicklung der Funktion $g_{x}$

$$
I_{2 x}=\int h_{x}(f) \exp \left\{-x^{2} a_{x} / 2-x^{2}\left(f-f_{0 x}\right) g_{x}{ }^{\prime \prime}(f)\left(f-f_{0 x}\right)^{T}(1+\dot{o}(1)) / 4\right\} d f \ldots
$$

Fiir alle $f \in \mathfrak{B}_{x}(A(x)) \backslash \Omega\left(f_{0 x}\right)$ gilt $\left(f-f_{0 x}\right) g_{x}{ }^{\prime \prime}(f)\left(f-f_{0 x}\right)^{T}>\lambda_{x}(f) \delta^{2}(\dot{x})$. Deshalb und wegen $h_{x}(f) \geqq 0$ gilt

$$
I_{2 x} \leqq \exp \left\{-x^{2} a_{x} / 2-x^{2}(1+o(1)) \lambda_{x}(f) \delta^{2}(x) / 4\right\} . \int h_{x}(f) d f .
$$

Aus ( $(5)$ und den Eigenschaften der Funktionen $\varrho$ und $\delta$ folgt schließlich

$$
I_{2 x}=o(1) x^{-1-k} \exp \left\{-x^{2} a_{x} / 2\right\}, \quad x \rightarrow \infty .
$$

Aus $(\Omega 1)$, : ( 2 2) und $(\Omega 6)$ folgt, daß gleichmäßig bezüglich $f \in \Omega\left(f_{0 x}\right)$ gilt $h_{x}(f)$ $=h_{x}\left(f_{0 x}\right)(1+o(1)), x \rightarrow \infty$. Das gestattet es, [4: Satz 4.6] anzuwenden, wobei die dort auftretende Funktion. $\mu$ in der Form $\mu(x)=\delta(x)\left(x^{2} \lambda_{x}(f) / 2\right)^{1 / 2}$ gewählt-werden kann. Es ergibt sich damit für $x \rightarrow \infty$

$$
\Phi(x A(x))=\left(1+O\left(1 / x^{2}\right)\right)\left((2 \pi)^{k} \operatorname{det} B(x)\right)^{-1 / 2} x^{k-2} I_{1 x},
$$


wobei

ist

$$
I_{1 x}=(1+o(1)) \frac{\pi^{(k-1) / 2} 2^{k-1} h\left(j f_{0 x}\right)}{\left(\operatorname{det} g_{x}{ }^{\prime \prime}\left(f_{0 x}\right)\right)^{1 / 2}} x^{1-k} \exp \left\{-x^{2} a_{x} / 2\right\}
$$

In dem Spezialfall, daß sowohl $R_{x}(\cdot)$ als auch $B(x)$ nicht von $x$ abhängen, sind die Voraussetzungen von Satz 1, d. h. $A \in \mathfrak{S} \mathfrak{B}$ mit $(\mathfrak{B} \dot{3})-(\mathfrak{B} 5)$ hinreichend für die Erfüllung der Voraussetzungen von Sat'z 3. In den oben angeführten Beispielen 1 und 2 ist dies der Fall, und aus Satz 3 folgen für diese Beispiele die Beziehungen

$$
\Phi(x A)=(1+o(1)) \cdot 2 \cdot(2 / \pi)^{1 / 2} x^{-1} \exp \left\{-x^{2} / 2\right\}, \quad x \rightarrow \infty
$$

bzw.

$$
\Phi(x A)=(1+o(1)) \cdot \cdot 3 \cdot(2 / \pi)^{1 / 2} x^{-1} \exp \left\{-x^{2} / 2\right\}, \quad x \rightarrow \infty,
$$

welche Präzisierungen von (9) hinsichtlich der Potenz.von $x$ und der Konstanten darstellen.

\section{LITERATUR}

[1] Eutrs, R. S., and J. S. Rosex: Laplace's method for Gaussian integrals with an application to Statistical Mechanics. Ann. Prob. 10 (1982), 46-66.

[2] Ellis, R. S., and J.S. Rośen! Asymptotic analysis of Gaussian integrals. I: Isolated minimum points. Trans. Amer. Math. Soc. 273 (1982), 447-481.

[3] Ellis, R. S., and J.S. Rosev: Asymptotic analysis of Gaussian integrals. II: Manifold of minimum points. Comm. Math. Phys. 82 (1981), 153-181.

[4] Федорюк, М. В.: Метоц перевала. Москва: Изд-во Наука 1977.

[5] МАслов, В. II., и॰М. В. Федорюк: Логарифмическая асимптотина интегралов Лапласа. Мат. заметки 30 (1981), 763-768.

[6] R.chter, W.-D.: Moderate deviations in' special sets of $\mathbf{R}^{k}$. Math. Nachr. 113 (1983), $339-354$.

[7] R.ICnTer, W.-D.: Laplice-Gauss-integrals, Gaussian mèasure asymptotic behaviour and probabilities of moderate deviations. Z. Anal. Anw. 4 (1985), 257-267.

[8] Richter, W.-D.: Remark on moderate deviations in the multi-dimensional central limit theorem. Math. Nachr. (in print).

[9] Рихтер, В.-Д.:. Интеграл Лапласа и вероятности умеренных уклонений в $\mathbf{R}^{k}$ : В сб: Труды III-ей Ферганской конф. по теории вероятн. и мат. стат. (в печати).

Manuskripteingang: 15. 11. 1983; in revidierter Fassung: 17.04. 1984

\section{VERFASSER :}

Hartmot Birind und Dr. Wolf-Dieter Rrchter

Sektion Mathematik der Technischen Universität

DDR-8027 Dresden, Mommsenstr. 13 